\title{
Eficácia e segurança dos antipsicóticos atípicos nas demências: uma revisão sistemática
}

\author{
Efficacy and safety of atypical antipsychotics in dementia: a sistematic review
}

\author{
Melissa Guarieiro Ramos e Fábio Lopes Rocha
}

\section{Resumo}

Objetivo: 0 emprego de antipsicóticos atípicos (AA) no tratamento de sintomas psicológicos e comportamentais das demências (SPCD) tem sido alvo de discussão em relação à eficácia e à segurança. 0 objetivo deste artigo é propiciar atualização sobre o tema. Métodos: Revisão da literatura publicada nos últimos dez anos com ênfase em metanálises e ensaios clínicos randomizados (ECR) controlados com placebo. Resultados: Três metanálises e nove ensaios clínicos foram analisados. Há evidências de eficácia clínica para risperidona ( $1 \mathrm{mg} / \mathrm{dia}$ ), olanzapina (5 a $10 \mathrm{mg} / \mathrm{dia}$ ) e aripiprazol (2 a 15mg/dia) no tratamento de agressividade e/ou SPCD em geral, e para risperidona ( $1 \mathrm{mg} / \mathrm{dia})$ no tratamento de sintomas psicóticos associados à demência. Os eventos adversos comuns com o uso de AA foram sonolência, sintomas extrapiramidais (SEP), incontinência ou infecção do trato urinário e alterações de marcha. $O$ tratamento com AA associou-se a maior risco de eventos cerebrovasculares e de mortalidade em idosos com demência. Conclusão: Baixas dosagens de risperidona, olanzapina e aripiprazol são eficazes na redução de agressividade e/ou SPCD globais; risperidona é eficaz na redução de sintomas psicóticos associados à demência. Em virtude de esses tratamentos associarem-se a pequeno aumento no risco de eventos cerebrovasculares e mortalidade, seu uso deve ser reservado para sintomatologia moderada/grave.

Palavras-chave: demência, antipsicóticos atípicos, sintomas neuropsiquiátricos.

\begin{abstract}
Objective: Concerns have been raised about efficacy and adverse events of atypical antipsychotics in the treatment of behavioural and psychological symptoms of dementia (BPSD). This paper is an update on current evidence of this theme. Methods: Review of published meta-analysis and randomized placebo-controlled trials (RCTs) in the last ten years. Results: Three meta-analysis and nine RCTs were evaluated. Evidence suggests that risperidone ( $1 \mathrm{mg} / \mathrm{day}$ ), olanzapine (5 to $10 \mathrm{mg} / \mathrm{day}$ ), and aripiprazole (2 to $15 \mathrm{mg} /$ day) are effective in treating aggression and/or BPSD overall; risperidone ( $1 \mathrm{mg} /$ day) reduces psychosis. Adverse events were mainly somnolence, extrapyramidal symptoms, urinary tract infection or incontinence, and abnormal gait with drug treatment. Atypical antipsychotics were associated with increased risk for cerebrovascular adverse events and mortality in elderly patients with dementia. Conclusion: Low doses of risperidone, olanzapine, and aripiprazole are effective in treating aggression and/or BPSD overall, and risperidone reduces psychosis associated with dementia. In view of the increased risk of cerebrovascular adverse events and mortality, the use of atypical antipsychotics in individuals with dementia should be reserved for patients with moderate/severe behavioural symptoms.
\end{abstract}

Key words: dementia, atypical antipsychotics, neuropsychiatric symptoms.
Recebido

07-08-06

Aprovado

21-09-06 


\section{Introdução}

A demência é uma síndrome caracterizada por declínio persistente em múltiplos domínios cognitivos. Associa-se a esse comprometimento cognitivo uma ampla gama de sintomas psiquiátricos, como alucinações, delírios, ansiedade, agressividade, agitação, comportamento excessivamente desinibido ou passivo, distúrbios do sono e/ou do humor, conhecidos como sintomas psicológicos e comportamentais das demências (SPCD) (McKeith e Cummings), 2005; Finkel et al., 1996; Cohen-Mansfield e Billing, 1998). Os SPCD ocorrem em 60\% a 90\% dos idosos com demência (Hope et al., 1999; Lyketsos et al., 2002).

O impacto negativo dessas alterações evidencia-se na piora da capacidade funcional e da qualidade de vida dos pacientes e por sofrimento e estresse dos familiares e cuidadores. Além disso, os SPCD contribuem para maior número de intervenções médicas, elevação dos custos (Helmels et al., 2001) e institucionalização prematura (Cohen et al., 1993).

Dentro do espectro dos SPCD destacam-se delírios, alucinações e comportamento agitado ou agressivo, que acometem $18 \%, 11 \%$ e $30 \%$ dos idosos com demência, respectivamente (Lyketsos et al., 2002). Para o manejo desses sintomas são utilizadas estratégias não-farmacológicas (Teri et al., 2002) e farmacológicas (Tariot et al., 2001). Entre essas, os antipsicóticos são os fármacos mais estudados (Herrmann, 2001; AGS/AAGP, 2003; Alexopoulos et al., 2004).

Até meados de 1990, os antipsicóticos típicos eram os fármacos mais empregados. Uma metanálise de sete ensaios controlados com placebo (Schneider et al., 1990) demonstrou eficácia de $18 \%$. Não se encontrou diferença em meio aos diversos antipsicóticos clássicos avaliados. Entre eles, o haloperidol foi o mais estudado e sua ação restringiu-se ao controle da agressividade (Lonergan et al., 2002). Além da modesta eficácia, os antipsicóticos típicos associaram-se a relevante incidência de sintomas extrapiramidais, discinesia tardia e efeitos anticolinérgicos.

$\mathrm{Na}$ última década, os antipsicóticos atípicos têm sido amplamente usados no tratamento dos SPCD (Alexopoulos et al., 2004; Lee et al., 2004; Sink et al., 2005; Carson et al., 2006), em virtude da menor incidência de sintomas extrapiramidais e discinesia tardia (Jeste et al., 1999a; Jeste et al., 1999b; van lersel et al., 2005). Apesar da rápida aceitação clínica, evidências relativas à sua eficácia e à sua segurança em SPCD são ainda limitadas. Particularmente, a possível associação entre o uso desses agentes e maior risco de mortalidade e eventos cerebrovasculares em idosos com demência tem sido alvo de discussões na comunidade médica. Este estudo é uma atualização sobre o tema e suas implicações na prática clínica.

\section{Métodos}

Foi realizada uma revisão da literatura indexada nas bases de dados MEDLINE, LILACS e da Biblioteca Cochrane no período de janeiro de 1996 a junho de 2006. Foram utilizados os seguintes termos de busca: dementia, neuropsychiatric symptoms e atypical antipsychotics. Foram incluídas metanálises e ensaios clínicos randomizados (ECR) controlados com placebo que avaliassem antipsicóticos atípicos (aripiprazol, clozapina, olanzapina, quetiapina, risperidona e ziprasidona) para terapêutica de SPCD.

\section{Resultados}

Foram encontradas três metanálises que avaliaram ECR, publicados e não-publicados. Duas metanálises, a de Ballard envolvendo dez ECR e a de Schneider envolvendo 15 ECR (Ballard e Waite, 2006 e Schneider et al., 2006a), avaliaram a eficácia e os eventos adversos dos antipsicóticos atípicos no tratamento de agressividade, agitação e sintomas psicóticos associados às demências. A terceira metanálise (15 ECR) (Schneider et al., 2005) enfocou o risco de morte associado ao uso de antipsicóticos atípicos no tratamento de SPCD.

Foram identificados nove ECR controlados com placebo, sendo quatro estudos com risperidona (De Deyn et al., 1999; Katz et al., 1999; Brodaty et al., 2003; Mintzer et al., 2006), dois com olanzapina (Street et al., 2000; De Deyn et al., 2004), um com risperidona e olanzapina (Debert et al., 2005), um com quetiapina (Ballard et al., 2005) e um com aripiprazol (De Deyn et al., 2005). Todos os ECR identificados foram incluídos nas metanálises, e suas características estão descritas na Tabela 1.

Sintetizamos a seguir as evidências relativas à eficácia e à segurança dos antipsicóticos atípicos.

\section{Eficácia dos antipsicóticos atípicos}

\section{Risperidona}

Há cinco ECR publicados e incluídos nas metanálises de Ballard e Waite (2006) e Schneider et al. (2006a). Foram avaliados 1.141 pacientes em uso de risperidona versus 755 com placebo por período de oito a 12 semanas de tratamento. Dos cinco ECR, dois (Katz et al., 1999; Brodaty et al., 2003) demonstraram eficácia da risperidona.

Katz et al. (1999) avaliaram a eficácia de doses fixas de risperidona (0,5; 1 ou $2 \mathrm{mg} / \mathrm{dia})$ em 625 idosos com demência, institucionalizados, com sintomas psicóticos ou comportamentais. Após 12 semanas, $45 \%$ dos pacientes que receberam risperidona $1 \mathrm{mg} / \mathrm{dia}(p=0,02)$ e $50 \%$ dos que receberam risperidona $2 \mathrm{mg} / \mathrm{dia}(p=0,002)$, comparados com $33 \%$ no grupo placebo, tiveram redução $\geq 50 \%$ na pontuação total da escala Behavioural Pathology in Alzheimer's Disease (BEHAVE-AD). A dose de $2 \mathrm{mg} /$ dia foi igualmente eficaz à dose de $1 \mathrm{mg} / \mathrm{dia}$ de risperidona e resultou em efeitos colaterais mais freqüentes, como sonolência e sintomas extrapiramidais.

No ensaio duplo-cego de De Deyn et al. (1999) com duração de 12 semanas, envolvendo 344 idosos institucionalizados, a risperidona (doses flexíveis: 0,5 a $4 \mathrm{mg} / \mathrm{dia}$; dose média: $1,1 \mathrm{mg} / \mathrm{dia}$ ) não apresentou resposta superior à do haloperidol (doses flexíveis: 0,5 a $4 \mathrm{mg} / \mathrm{dia}$; dose média: $1,2 \mathrm{mg} / \mathrm{dia}$ ) ou à do placebo na avaliação primária de eficácia (redução $\geq 30 \%$ BEHAVE-AD).

Brodaty et al. (2003) avaliaram os efeitos da risperidona (doses flexíveis: 0,5 a $2 \mathrm{mg} / \mathrm{dia}$ ) no tratamento de 345 idosos institucionalizados e com comportamento agressivo 
Ramos MG \& Rocha FL

Tabela 1. Características dos ensaios clínicos publicados duplo-cegos, randomizados e controlados com placebo

\begin{tabular}{|c|c|c|c|c|c|}
\hline Estudo & Intervenções (mg/dia) & $\begin{array}{l}\text { Duração } \\
\text { (semanas) }\end{array}$ & Participantes & $\begin{array}{l}\text { Eficácia } \\
\text { primária/resultados }\end{array}$ & Eventos adversos \\
\hline \multicolumn{6}{|l|}{ Risperidona } \\
\hline Katz et al., 1999 & 0,$5 ; 1$ ou 2 & 12 & $\begin{array}{l}n=625 ; \text { demência } \\
\text { com SPCD (73\% DA, } \\
15 \% \text { DV, } 12 \% \text { DM); } \\
68 \% \text { \%; idade média: } \\
83 \text { anos; MMSE } \\
\text { (média): } 6,6 ; \text { inst. }\end{array}$ & $\begin{array}{l}\text { BEHAVE-AD (redução } \\
\geq 50 \%) ;(+ \text { risperi- } \\
\text { dona }(1 \text { e } 2 \mathrm{mg} / \mathrm{d})> \\
\text { placebo }\end{array}$ & $\begin{array}{l}\text { Sonolência e SEP: } \\
\text { risperidona > placebo } \\
\text { (dose-depedente) }\end{array}$ \\
\hline De Deyn et al., 1999 & $\begin{array}{l}\text { 0,5-4, dose média: } 1,1 \\
\text { vs. haloperidol 0,5-4, } \\
\text { dose média: } 1,2\end{array}$ & 12 & $\begin{array}{l}n=344 ; \text { demência } \\
\text { com SPCD (67\% DA, } \\
26 \% \text { DV, } 7 \% \text { DM); } \\
56 \% \text { o; idade média: } \\
\text { 81,3 anos; MMSE } \\
\text { (média): 8,7; Inst. }\end{array}$ & $\begin{array}{l}\text { BEHAVE-AD (redução } \\
\geq 30 \% \text { ); risperidona }= \\
\text { placebo = haloperidol }\end{array}$ & $\begin{array}{l}\text { Sonolência: risperido- } \\
\text { na > placebo; SEP: } \\
\text { haloperidol > risperi- } \\
\text { dona = placebo }\end{array}$ \\
\hline Brodaty et al., 2003 & 0,5-2, dose média: 0,95 & 12 & $\begin{array}{l}n=345 ; \text { demência } \\
\text { com agressividade } \\
\text { (58\% DA, } 29 \% \text { DV, } \\
13 \% \text { DM); } 71 \% \text {; } \\
\text { idade média: } 83 \text { anos; } \\
\text { MMSE (média): } 5,5 ; \\
\text { inst. }\end{array}$ & $\begin{array}{l}\text { CMAI (subescala de } \\
\text { agressividade) }(+) \\
\text { risperidona > placebo }\end{array}$ & $\begin{array}{l}\text { Sonolência e eventos } \\
\text { adversos graves (5 } \\
\text { AVCs e } 1 \text { AIT) no gru- } \\
\text { po com risperidona }\end{array}$ \\
\hline Debert et al., 2005 & $\begin{array}{l}\text { 1,5-2, dose média: } 1 \text { vs. } \\
\text { olanzapina } 2,5-10 \text {, dose } \\
\text { média: } 5,2\end{array}$ & 10 & $\begin{array}{l}n=494 \text {; demência } \\
\text { com sintomas psicóti- } \\
\text { cos ( } 82 \% \text { DA, } 6 \% \text { DV, } \\
13 \% \text { DM); } 66 \% \text {; ; ida- } \\
\text { de média: } 78,5 \text { anos; } \\
\text { MMSE (média): } 14,4 ; \\
\text { inst. e ambulatoriais }\end{array}$ & $\begin{array}{l}\mathrm{NPI} / \mathrm{NH} \text { - soma } \\
\text { alucinações e delírios } \\
\text { e CGI-S Psychosis; } \\
\text { risperidona = olanza- } \\
\text { pina = placebo }\end{array}$ & $\begin{array}{l}\text { Sonolência/inconti- } \\
\text { nência urinária/hosti- } \\
\text { lidade: olanzapina = } \\
\text { risperidona > placebo }\end{array}$ \\
\hline Mintzer et al., 2006 & $0,5-1,5$, dose média: 1,03 & 8 & $\begin{array}{l}n=473 ; \text { DA com } \\
\text { sintomas psicóticos; } \\
77 \% \text { o; idade média: } \\
83,3 \text { anos; MMSE } \\
\text { (média): 13,2; inst. }\end{array}$ & $\begin{array}{l}\text { BEHAVE-AD } \\
\text { (subescala de } \\
\text { psicose) e CGI-C; } \\
\text { risperidona = placebo }\end{array}$ & $\begin{array}{l}\text { Risperidona: > sono- } \\
\text { lência, SEP; } 9 \text { mortes } \\
\text { (3,8\%); } 3 \text { AIT e } 1 \text { AVC }\end{array}$ \\
\hline \multicolumn{6}{|l|}{ Olanzapina } \\
\hline Street et al., 2000 & 5,10 ou 15 & 6 & $\begin{array}{l}n=206 \text {; DA com } \\
\text { agitação, delírios ou } \\
\text { alucinações; } 61 \% \text {; } \\
\text { idade média: } 82,8 \\
\text { anos; MMSE (média): } \\
6,9 \text {; inst. }\end{array}$ & $\begin{array}{l}\mathrm{NPI} / \mathrm{NH} \text { - soma agi- } \\
\text { tação/agressividade/ } \\
\text { alucinações e delírios } \\
\text { (redução } \geq 50 \% \text { ); } \\
\text { (+) olanzapina (5 e } \\
\text { 10mg/d) > placebo }\end{array}$ & $\begin{array}{l}\text { Sonolência ( } 5 \text { a } 8 x \text { ) e } \\
\text { distúrbio de marcha } \\
\text { ( } 7,5 \text { a } 11 x)>\text { com } \\
\text { olanzapina; SEP: } \\
\text { olanzapina = placebo }\end{array}$ \\
\hline De Deyn et al., 2004 & $1 ; 2,5 ; 5 ; 7,5$ & 10 & $\begin{array}{l}n=652 ; \text { DA com alu- } \\
\text { cinações ou delírios; } \\
75 \% \text {; ; idade média: } \\
76,6 \text { anos; MMSE } \\
\text { (média): } 13,7 ; \text { inst. }\end{array}$ & $\begin{array}{l}\mathrm{NPI} / \mathrm{NH} \text { (subescala } \\
\text { de psicose) e CGI-C; } \\
\text { olanzapina = placebo }\end{array}$ & $\begin{array}{l}\text { Olanzapina: > ganho } \\
\text { de peso, anorexia e } \\
\text { incontinência urinária }\end{array}$ \\
\hline \multicolumn{6}{|l|}{ Aripiprazol } \\
\hline De Deyn et al., 2005 & 2-15, dose média: 10 & 10 & $\begin{array}{l}n=208 ; \text { DA com } \\
\text { sintomas psicóticos; } \\
72 \% \text { o; idade média: } \\
81,5 \text { anos; MMSE } \\
\text { (média): } 14,2 ; \\
\text { ambulatoriais }\end{array}$ & $\begin{array}{l}\text { NPI (subescala de } \\
\text { psicose); aripiprazol = } \\
\text { placebo }\end{array}$ & $\begin{array}{l}\text { Aripiprazol: > lesões } \\
\text { acidentais, sonolência }\end{array}$ \\
\hline \multicolumn{6}{|l|}{ Quetiapina } \\
\hline Ballard et al., 2005 & $\begin{array}{l}\text { 50-100, dose média: } 94 \\
\text { vs. rivastigmina } 6-12 \text {, } \\
\text { dose média: } 8,4\end{array}$ & 26 & $\begin{array}{l}n=80 ; \text { DA com } \\
\text { agitação; } 80 \% \text {; } \\
\text { idade média: 83,8 } \\
\text { anos; MMSE (média): } \\
\text { NR; inst. }\end{array}$ & $\begin{array}{l}\mathrm{CMAl} \text {; quetiapina = } \\
\text { rivastigmina = placebo }\end{array}$ & $\begin{array}{l}\text { Quetiapina: > declínio } \\
\text { cognitivo }\end{array}$ \\
\hline
\end{tabular}

SPCD: sintomas psicológicos e comportamentais das demências; DA: demência de Alzheimer; DV: demência vascular; DM: demência mista; inst.: institucionalizados; MMSE: Mini-Mental State Examination; BEHAVE-AD: Behavioural Pathology in Alzheimer's Disease rating scale; CMAl: Cohen-Mansfield Agitation Inventory; NPI/NH: Neuropsychiatric Inventory/Nursing Home version; CGI-C: Clinical Global Impression of Change; CGI-S Psychosis: Clinical Global Impression Severity of Psychosis Scale; SEP: sintomas extrapiramidais; AVC: acidente vascular cerebral; AIT: ataque isquêmico transitório; NR: não-relatado. 
significativo, por período de 12 semanas. A risperidona (dose média: 0,95mg/dia) apresentou eficácia superior ao placebo $(p<0,001)$ na redução na pontuação da subescala de agressividade da Cohen-Mansfield Agitation Inventory (CMAI).

Em 2005, Debert et al. compararam a eficácia da risperidona (dose média: $1 \mathrm{mg} / \mathrm{dia} ; n=196$ ) versus olanzapina e placebo no controle de sintomas psicóticos de 494 idosos com demência, por período de dez semanas. Não houve diferenças estatisticamente significativas entre os grupos na redução na soma dos itens alucinações e delírios da escala Neuropsychiatric Inventory (NPI) e da Clinical Global Impression/Severity of Psychosis (CGI-S Psychosis).

Em estudo recente, Mintzer et al. (2006) avaliaram a eficácia e a segurança da risperidona (doses flexíveis: 1 a $1,5 \mathrm{mg} / \mathrm{dia}$ ) no tratamento de sintomas psicóticos na doença de Alzheimer (DA) $(n=473)$ por oito semanas. Não houve diferença estatisticamente significativa entre risperidona (dose média: $1,03 \mathrm{mg} / \mathrm{dia}$ ) e placebo na redução na subescala de sintomas psicóticos da BEHAVE-AD e na Clinical Global Impression of Change (CGI-C).

Nas metanálises de Ballard e Waite (2006) e de Schneider et al. (2006), a risperidona (1 e $2 \mathrm{mg} / \mathrm{dia}$ ) apresentou eficácia global nas subescalas de sintomas psicóticos e agressividade. Em todos os ECR houve elevadas taxas de resposta $(33 \%-66,2 \%)$ no grupo placebo.

\section{Olanzapina}

Existem três ECR publicados. No total foram avaliados 876 pacientes em uso de olanzapina e 270 pacientes com placebo, por período de seis a dez semanas. Em apenas um ECR (Street et al., 2000) a olanzapina apresentou eficácia superior à do placebo. Street et al. (2000) avaliaram a eficácia da olanzapina em 206 idosos com DA, por seis semanas. Doses de 5 e $10 \mathrm{mg} / \mathrm{dia}$, e não $15 \mathrm{mg} / \mathrm{dia}$, foram significativamente superiores ao placebo na redução de pelo menos $50 \%$ na pontuação da soma de três sintomas nucleares (agitação/ agressividade, alucinações e delírios) da Neuropsychiatric Inventory/Nursing Home version (NPI/NH).

De Deyn et al. (2004) avaliaram a eficácia de doses fixas de olanzapina $(1 ; 2,5 ; 5$ ou $7,5 \mathrm{mg} / \mathrm{dia})$ no tratamento de sintomas psicóticos de 652 idosos durante dez semanas. Não houve diferença entre os grupos olanzapina e placebo.

No ensaio de Debert et al. (2005) foi comparada a eficácia de doses flexíveis de olanzapina (dose média: $5,2 \mathrm{mg} / \mathrm{dia} ; n=204$ ) versus risperidona e placebo no controle de sintomas psicóticos em 494 idosos durante dez semanas. Não houve diferença de eficácia entre os grupos.

$\mathrm{Na}$ metanálise de Schneider et al. (2006a) foram incluídos os três ECR citados. Entre esses ensaios, não foi encontrada eficácia global após análise combinada nas escalas NPI e Brief Psychiatric Rating Scale (BPRS). Entretanto na metanálise de Ballard e Waite (2006) foram incluídos os três ECR citados e um ECR não-publicado, cujo resultado era positivo. Após análise combinada dos dados segregados em subescalas, a olanzapina (5 a $10 \mathrm{mg} / \mathrm{dia}$ ) demonstrou eficácia na redução da agressividade. Em todos os ensaios houve elevada resposta ( $33 \%$ a $66,2 \%)$ com placebo.

\section{Aripiprazol}

Há um ECR publicado. De Deyn et al. (2005) compararam aripiprazol (2 a $15 \mathrm{mg} / \mathrm{dia}$ ) com placebo no tratamento de sintomas psicóticos em idosos com DA em 208 pacientes durante dez semanas. 0 aripiprazol foi similar ao placebo $(p=0,17)$ na medida de eficácia primária (redução na subescala de psicose da NPI).

Na metanálise de Ballard e Waite (2006), o ECR citado foi o único incluído. Schneider et al. (2006a) incluíram em sua metanálise o estudo de De Deyn et al. (2005) e dois ECR não-publicados. Esses ensaios utilizaram desenhos similares e analisaram idosos com DA e sintomas psicóticos por período de dez semanas. 0 aripiprazol (2 a 15mg/dia) demonstrou eficácia global significativa, sem eficácia no controle específico de sintomas psicóticos.

\section{Quetiapina}

Há um ECR publicado. Ballard e Waite (2005) investigaram a eficácia da quetiapina e da rivastigmina no controle da agitação associada à DA e avaliaram suas repercussões também no desempenho cognitivo ( $n=80$; duração: 26 semanas). Nem a quetiapina (doses flexíveis: 50 a $100 \mathrm{mg} / \mathrm{dia}$ ) nem a rivastigmina (doses flexíveis: 6 a 12mg/dia), em comparação com o placebo, resultaram em melhora significativa. A quetiapina associou-se a declínio cognitivo superior ao placebo.

Na metanálise de Ballard e Waite (2006), o ECR citado foi o único incluído. Não houve dados suficientes para avaliação de eficácia. Schneider et al. (2006a) incluíram em sua metanálise o estudo de Ballard et al. (2005) e dois ECR não-publicados, também sem diferença significativa entre quetiapina e placebo. Em virtude da heterogeneidade dos ECR, não foi possível a análise combinada dos resultados de eficácia.

\section{Eventos adversos comuns e abandonos}

Nos ensaios, os eventos adversos foram relatados heterogeneamente e de forma incompleta. Sonolência, quedas, lesões (sem especificação), síncope, sintomas extrapiramidais, hematomas (equimoses, púrpuras e contusões), edema e infecções do trato urinário foram os mais freqüentemente mencionados (van lersel et al., 2005; Ballard e Waite, 2006; Schneider et al., 2006a).

Segundo metanálise de Schneider et al. (2006a), os sintomas mais freqüentes dos fármacos estudados foram sonolência (dose-dependente) (odds ratio $[\mathrm{OR}]=2,84$; IC 95\%: 2,25-3,58), infecção do trato urinário e incontinência urinária (OR = 1,51; IC 95\%: 1,07-2,12) e sintomas extrapiramidais $(\mathrm{OR}=1,51 ; \mathrm{IC} 95 \%: 1,2-1,91)$.

Entre todos os antipsicóticos atípicos, a risperidona associou-se mais fortemente a sintomas extrapiramidais (OR = 1,8; IC 95\%: 1,35-2,42). Risperidona e olanzapina associaram-se mais comumente a anormalidades de marcha (OR = 3,42; IC 95\%: 1,78-6,56). Declínio cognitivo associouse significativamente ao uso de antipsicóticos atípicos em comparação ao placebo $(p<0,0001)$.

Um total de $1.079(32,2 \%)$ pacientes nos grupos em tratamento e $551(31,4 \%)$ pacientes no grupo placebo abandonou os estudos por causas diversas, embora com significativa heterogeneidade entre os ensaios (Schneider et al., 2005). 


\section{Risco de eventos cerebrovasculares e mortalidade}

0 estudo de Brodaty et al. (2003) foi o primeiro a relatar aumento na incidência de eventos cerebrovasculares $e$ maior mortalidade entre idosos com demência tratados com risperidona. Nesse ensaio, 16,8\% dos pacientes em uso de risperidona (versus $8,8 \%$ no grupo placebo) apresentaram eventos adversos graves, incluindo cinco acidentes cerebrovasculares e um ataque isquêmico transitório. Dez pacientes faleceram: seis $(3,6 \%)$ no grupo da risperidona e quatro $(2,4 \%)$ no do placebo. As causas mais freqüentes foram pneumonia (três com risperidona e um com placebo) e acidente vascular cerebral (AVC) (dois com risperidona).

Revisão de ECR não-publicados, conduzida pela Food and Drug Administration (FDA) e Health Canada, também apontou maior mortalidade associada ao uso de antipsicóticos atípicos em SPCD (FDA Public Health Advisory, 2005). Esses órgãos analisaram retrospectivamente $17 \mathrm{ECR}$ que envolveram 5.106 idosos portadores de demência em uso de placebo ou de um dos quatro atípicos (olanzapina, risperidona, aripiprazol e quetiapina), por período de até dez semanas de tratamento. Os dados demonstraram aumento de 1,7 vez no risco relativo de morte, com risco absoluto de 1,9\% entre os idosos em uso de antipsicóticos de segunda geração (Singh e Wooltorton, 2005). A maioria das mortes relatadas deveu-se a eventos cardíacos (morte súbita e insuficiência cardíaca) ou a infecções (principalmente pneumonia).

Desde então a constatação desses eventos adversos graves em estudos similares com risperidona e olanzapina justificou a divulgação de alertas de segurança (Wooltorton, 2002 e 2004).

Consubstancia esses dados a metanálise realizada por Schneider et al. (2005) com 15 ECR (nove não-publicados) para avaliar o risco de morte associado ao uso de antipsicóticos atípicos em demência. Esses estudos, com duração de oito a 12 semanas, evidenciaram pequeno aumento no risco de mortalidade, 3,5\% de mortes com antipsicóticos atípicos e 2,3\% com placebo (OR = 1,54; IC 95\%: 1,06-2,23). O risco só pôde ser identificado quando os resultados foram combinados. Expressos em risco de morte por paciente, esses resultados significam que os antipsicóticos atípicos potencialmente causam uma morte a cada 85 pacientes tratados e, se extrapolados em mortes de pessoas por ano de tratamento, significam uma morte a cada 12 pacientes por ano de tratamento (Schneider et al., 2006b).

Maior risco de mortalidade também parece se associar ao uso de antipsicóticos típicos comparativamente aos atípicos, conforme 0 estudo de Wang et al. (2005). Esses autores conduziram estudo de coorte retrospectivo com 22.890 indivíduos ( $\geq 65$ anos) que iniciaram uso de antipsicóticos convencionais ou atípicos entre 1994 e 2003. Foram comparados os riscos de mortalidade em diferentes intervalos em até 180 dias de terapia com antipsicóticos. Os antipsicóticos convencionais se associaram a risco ajustado significativamente maior de morte em comparação aos atípicos para todos os intervalos estudados, com riscos maiores logo após o início da terapia ( $<40$ dias) e com doses mais altas dos fármacos.

Quanto ao risco de eventos cerebrovasculares, conforme metanálise de Schneider et al. (2006a), há evidências de que os antipsicóticos atípicos (1,9\%) associam-se a maior risco em comparação ao placebo $(0,9 \%)(\mathrm{OR}=2,13$; IC $95 \%$ : 1,2-3,75). Maior risco de eventos cerebrovasculares parece se associar similarmente ao uso de antipsicóticos típicos (Gill et al., 2005; Percudani et al., 2005), embora os ensaios iniciais tenham apresentado evidências controversas e não-favoráveis a esse potencial risco (Herrmann et al., 2004; Herrmann et al., 2005; Liperoti et al., 2006).

\section{Discussão}

Na maioria dos ECR foram avaliados idosos em sua oitava década de vida, do sexo feminino, portadores de DA (estágio moderado a grave), institucionalizados, por período médio de dez semanas de tratamento. As evidências demonstram eficácia modesta global da risperidona ( $1 \mathrm{mg} /$ dia) e específica no controle da agressividade e sintomas psicóticos associados às demências. A olanzapina ( 5 a 10mg/dia) mostrou eficácia apenas em agressividade e 0 aripiprazol (2 a 15mg/dia) apresentou redução global de sintomas, entretanto sem melhora de sintomas psicóticos. Para a quetiapina, as evidências são limitadas e não demonstraram eficácia superior à do placebo. Não há estudos controlados sobre o emprego de clozapina ou ziprasidona em SPCD.

$\mathrm{Na}$ interpretação dos resultados sobre eficácia devem ser consideradas algumas limitações metodológicas. Em primeiro lugar, há de se considerar a inclusão de diversos tipos de demência, em diferentes fases de evolução, com pacientes institucionalizados ou ambulatoriais, e a gama de sintomas psiquiátricos envolvidos. Além disso, diferentes estudos empregam distintas escalas (e/ou subescalas) e critérios para avaliar a eficácia dos tratamentos. Alguns ensaios abordam escores totais de escalas que abrangem diversos SPCD; outros avaliam alguns sintomas específicos como agitação, agressividade ou sintomas psicóticos. Essa heterogeneidade torna difícil a interpretação dos ensaios individualmente e sua combinação em estudos de metanálise. É possível que a dificuldade de evidenciar atividade antipsicótica de típicos e atípicos em pacientes com demência possa ser atribuída a esses problemas metodológicos. Outro problema é a transposição da significância estatística para efeitos clinicamente significativos.

Aspecto importante é a elevada resposta ao placebo observada $(33 \%-66,2 \%)$. Esse fato provavelmente reflete uma significativa taxa de resolução espontânea desses sintomas e aponta para um possível valor das co-intervenções nãofarmacológicas.

Os eventos adversos mais comuns com o uso de antipsicóticos atípicos foram sonolência, sintomas extrapiramidais, incontinência e/ou infecção do trato urinário. Entre os antipsicóticos atípicos, risperidona foi a mais freqüentemente associada a sintomas extrapiramidais; e risperidona e olanzapina, a alterações de marcha. Maior declínio cognitivo associou-se ao uso de antipsicóticos atípicos. Esses efeitos colaterais demonstraram-se dose-dependentes (van lersel et al., 2005), o que sustenta a prática de iniciar os antipsicóticos em baixas dosagens e progredir com ajustes graduais conforme a tolerância do paciente.

Ensaios clínicos de curto prazo (oito a 12 semanas) mostraram que o uso de antipsicóticos atípicos em idosos 
com demência associa-se a aumento aproximado de 1,5 vez no risco de mortalidade e de duas vezes no risco de eventos cardiovasculares. As hipóteses para o aumento de mortalidade são relacionadas aos efeitos colaterais dos antipsicóticos atípicos, como sedação, sintomas extrapiramidais e distúrbios de marcha. Esses efeitos colaterais aumentariam o tempo de permanência do paciente na cama com aumento do risco de infecção e de problemas respiratórios e cardiovasculares (Schneider et al., 2006a). Os mecanismos aventados para 0 maior risco de eventos cerebrovasculares são efeitos tromboembólicos, alterações cardiovasculares (arritmias e hipotensão ortostática), sedação excessiva (por resultar em desidratação e hemoconcentração) e hiperprolactinemia (por promover agregação plaquetária) (Herrmann et al., 2005). Embora não representem contra-indicação absoluta, esses dados modificam a análise de riscos e benefícios e reforçam a necessidade de controle clínico mais vigilante dos fatores de risco para eventos cardiovasculares nesses pacientes. Ressalte-se que o emprego de antipsicóticos típicos também está associado ao aumento do risco de mortalidade e eventos cerebrovasculares.

Na prática clínica, se as alterações comportamentais não se associam a ameaças sérias imediatas para o paciente ou terceiros, a abordagem desses sintomas deveria ser baseada principalmente na orientação aos cuidadores e familiares, em modificações ambientais e intervenções comportamentais, além da avaliação do estado clínico geral do paciente e tratamento dos problemas clínicos encontrados. 0 uso de antipsicóticos atípicos deve ser reservado para pacientes com agressividade, agitação ou sintomas psicóticos moderados a graves. Quando indicados, os antipsicóticos atípicos devem ser iniciados em doses baixas e titulados até a menor dose que seja eficaz e bem tolerada. Espera-se que a melhora

\section{Referências}

Alexopoulos GS, Streim J, Carpenter D, Docherty JP. Expert consensus guideline series: using antipsychotic agents in older patients. J Clin Psychiatry, 65(Suppl 2): 1-105, 2004.

American Geriatrics Society and American Association for Geriatric Psychiatry (AGS/AAGP). Consensus statement on improving the quality of mental health care in US nursing homes: management of depression and behavioural symptoms associated with dementia. J Am Geriatr Soc, 51: 1287-98, 2003.

Ballard C, Margallo-Lana M, Juszcak E, Douglas S, Swann A, Thomas A et al. Quetiapine and rivastigmine and cognitive decline in Alzheimer's disease: randomized double-blind placebo-controlled trial. BMJ, 330(7496): 874, 2005.

Ballard C, Waite J. The effectiveness of atypical antipsychotics for the treatment of aggression and psychosis in Alzheimer's disease. Cochrane Database Syst Rev. 2006 Jan 25; (1): CD003476.

Brodaty H, Ames D, Snowdon J, Woodward M, Kirwan J, Clarnette R et al. A randomized placebo-controlled trial of risperidone for the treatment of aggression, agitation, and psychosis of dementia. J Clin Psychiatry, 64: 134-43, 2003 ocorra em um período máximo de dez a 12 semanas, duração usual dos ensaios clínicos (Schneider et al., 2006a). Caso não ocorra, recomenda-se outra estratégia terapêutica incluindo intervenções não-farmacológicas. Avaliações clínicas freqüentes devem ser realizadas para eventuais ajustes de doses, modificação do fármaco ou para determinar a necessidade de continuar a medicação (NGSMH, 2006). Um aspecto usual na prática clínica e pouco evidenciado nos ensaios clínicos é a necessidade freqüente de troca de medicamentos em virtude da sensibilidade exacerbada desses pacientes ou do surgimento de efeitos paradoxais.

\section{Conclusão}

Sintomas psicóticos, agressividade e agitação associados à demência são responsáveis por substancial estresse para o paciente e seus cuidadores e são de difícil tratamento. Há evidências consistentes de que os antipsicóticos atípicos risperidona, olanzapina e aripiprazol têm eficácia modesta no controle da agressividade e/ou dos SPCD em geral. Apenas a risperidona demonstrou eficácia no controle de sintomas psicóticos. A maioria dos efeitos colaterais é dose-dependente. 0 uso desses fármacos também se associa a aumento de 1,5 vez no risco de mortalidade e de duas vezes no risco de eventos cardiovasculares em idosos com demência. Esse risco potencial indica a necessidade de cuidadosa avaliação de riscos e benefícios da utilização de atípicos nessa população.

Potenciais conflitos de interesse

O Dr. Fábio L. Rocha participa de ensaios clínicos das indústrias farmacêuticas Bristol-Myers Squibb Farmacêutica, Laboratórios Servier, GlaxoSmithKline, Pfizer.

Carson S, McDonagh MS, Peterson K. A systematic review of the efficacy and safety of atypical antipsychotics in patients with psychological and behavioural symptoms of dementia. J Am Geriatr Soc, 54(2): 354-61, 2006

Cohen CA, Gold DP, Shulman KI, Wortley JT, McDonald G, Wargon $M$. Factors determining the decision to institutionalize dementia individuals: a prospective study. Gerontologist, 33: 714-20, 1993.

Cohen-Mansfield J, Billing N. Agitated behaviours in the elderly. I: A conceptual review. J Am Geriatr Soc, 36: 7-12, 1998.

Debert WG, Dysken MW, Rappaport SA, Feldman PD, Young CA, Hay DP et al. Comparison of olanzapine and risperidone in the treatment of psychosis and associated behavioural disturbances in patients with dementia. Am J Geriatr Psychiatry, 13: 722-30, 2005.

De Deyn PP, Rabheru K, Rasmussen A, Bocksberger JP, Dautzenber $\mathrm{PLJ}$, Eriksson $\mathrm{S}$ et al. A randomized trial of risperidone, placebo, and haloperidol for behavioural symptoms of dementia. Neurology, 53: 946-55, 1999.

De Deyn PP, Carrasco MM, Debert W et al. Olanzapine versus placebo in the treatment of psychosis with or without associated behavioural 
disturbances in patients with Alzheimer's disease. Int J Geriatr Psychiatry, 19: 9-18, 2004.

De Deyn PP, Jeste DV, Swanink R, Kostic D, Breder C, Carson W, Iwamoto T. Aripiprazole for the treatment of psychosis in patients with Alzheimer's disease. J Clin Psychopharmacol, 25: 463-67, 2005.

FDAPublic Health Advisory. Deaths with antipsychotics in elderly patients with behavioural disturbances. U.S. Food and Drug Administration, 2005 Disponivel em: www.fda.gov/cder/drug/advisory/antipsychotics.htm.

Finkel SI, Costa e Silva J, Cohen G, Miller S, Sartorius N. Behavioural and psychological signs and symptoms of dementia: a consensus statement on current knowledge and implications for research and treatment. Int Psychogeriatr, 8(Suppl 3): 497-500, 1996.

Gill SS, Rochon PA, Herrmann N et al. Atypical antipsychotic drugs and risk of ischaemic stroke: population-based retrospective cohort study. BMJ, 330: 445, 2005.

Hemels MEH, Lanctôt KL, Iskedjian M, Einarson TR. Clinical and economic factors in the treatment of behavioural and psychological symptoms of dementia. Drugs Aging, 18(7): 527-50, 2001.

Herrmann N. Recommendations for the management of behavioural and psychological symptoms of dementia. Can J Neurol Sci 28(Suppl 1): 96-107, 2001.

Herrmann N, Mamdani M, Lanctôt KL. Atypical antipsychotics and risk of cerebrovascular accidents. Am J Psychiatry, 161: 1113-5, 2004.

Herrmann N, Lanctôt KL. Do atypical antipsychotics cause stroke? CNS Drugs, 19(2): 91-103, 2005.

Hope T, Keene J, Fairburn CG, Jacoby R, McShane R. Natural history of behavioural changes and psychiatric symptoms in Alzheimer's disease. A longitudinal study. Br J Psychiatry, 174: 39-44, 1999.

Jeste Dv, Rockwell E, Harris MJ, Lohr JB, Lacro JP. Conventional versus newer antipsychotics in elderly patients. Am J Geriatr Psychiatry, 7: 70-6, 1999a.

Jeste Dv, Lacro JP, Bailey A, Rockwell E, Harris MJ, Caligiuri MP. Lower incidence of tardive dyskinesia with risperidone compared with haloperidol in older patients. J Am Geriatr Soc, 47: 716-9, 1999b.

Katz I, Jeste DV, Mintzer JE, Clyde C, Napolitano J, Brecher M et al. Comparison of risperidone and placebo for psychosis and behavioural disturbances associated with dementia: a randomized, double-blind trial: Risperidone Study Group. J Clin Psychiatry, 60: 107-15, 1999.

Lee PE, Gill SS, Freedman M, Bronskill SE, Hillmer MP, Rochon PA Atypical antipsychotic drugs in the treatment of behavioural and psychological symptoms of dementia: systematic review. BMJ, 329: 75-80, 2004.

Liperoti R. Review: atypical antipsychotic drugs slightly increase mortality rates in elderly people with dementia. Evid Based Ment Health, 9(2): 54, 2006.

Lonergan E, Luxenberg J, Colford J. Haloperidol for agitation in dementia (Cochrane Review). In: The Cochrane Library, Issue 1, 2002. Oxford: Update Software.

Lyketsos CG, Lopez O, Jones B et al. Prevalence of neuropsychiatric symptoms in dementia and mild cognitive impairment: results from the Cardiovascular Health Study. JAMA, 288: 1475-83, 2002.

McKeith I, Cummings J. Behavioural changes and psychological symptoms in dementia disorders. Lancet Neurol, 4: 735-42, 2005.
Mintzer J, Greenspan A, Caers I, Hove IV, Kushner S, Weiner M et al. Risperidone in the treatment of psychosis of Alzheimer disease: results from a prospective clinical trial. Am J Geriatr Psychiatry, 14: 280-91, 2006

NGSMH. National Guidelines for Seniors' Mental Health. The assessment and treatment of mental health issues in long-term care homes (focus on mood and behavior symptoms). Canadian Coalition for Seniors' Mental Health. May, 2006. Disponivel em: http://www.ccsmh. ca/en/download.cfm.

Percudani M, Barbui C, Fortino I, Tansella M, Petrovich L. Secondgeneration antipsychotics and risk of cerebrovascular accidents in the elderly. J Clin Psychopharmacol, 25: 468-70, 2005.

Schneider LS, Pollock VE, Lyness SA. A meta-analysis of controlled trial of neuroleptic treatment in dementia. J Am Geriatr Soc, 38: 553-63, 1990.

Schneider LS, Dagerman KS, Insel P. Risk of death with atypical antipsychotic drug treatment for dementia. Meta-analysis of randomized placebo-controlled trials. JAMA, 294(15): 1934-43, 2005.

Schneider LS, Dagerman K, Insel PS. Efficacy and adverse events of atypical antipsychotics for dementia: meta-analysis of randomized, placebo-controlled trials. Am J Geriatr Psychiatry, 14(3): 191-210, $2006 a$.

Schneider LS, Dagerman K, Insel PS, Hirsch C. Review: atypical antipsychotic drugs increase risk for death in dementia. ACP Journal Club, 144: 2, 2006b.

Singh S, Wooltorton E. Increased mortality among elderly patients with dementia using atypical antipsychotics. CMAJ, 173(3): 252, 2005.

Sink KM, Holden KF, Yaffe K. Pharmacological treatment of neuropsychiatric symptoms of dementia: a review of the evidence. JAMA, 293(5): 596-608, 2005.

Street JS, Clarck WS, Gannon KS, Cummings JL, Bymaster FP, Tamura RN et al. Olanzapine treatment of psychotic and behavioural symptoms in patients with Alzheimer disease in nursing care facilities: a doubleblind, randomized, placebo-controlled trial. Arch Gen Psychiatry, 57: 968-76, 2000

Tariot PN, Ryan JM, Porstcinsson AP, Loy R, Schneider LS. Pharmacologic therapy for behavioural symptoms of Alzheimer's disease. Clin Geriatr Med, 17: 359-76, 2001.

Teri L, Logsdon RG, McCurry SM. Nonpharmacologic treatment of behavioural disturbance in dementia. Med Clin North Am, 86: 641-56, 2002.

van lersel MB, Zuidema SU, Koopmans RTCM, Verhey FRJ, Rikkert MGMO. Antipsychotics for behavioural and psychological problems in elderly people with dementia: a systematic review of adverse events. Drugs Aging, 22(10): 845-58, 2005.

Wang PS, Schneeweiss S, Avorn J, Fischer MA, Mogun H, Solomon $\mathrm{DH}$ et al. Risk of death in elderly users of conventional vs. atypical antipsychotic medications. N Engl J Med, 353: 2335-41, 2005.

Wooltorton E. Risperidone (Risperdal): increased rate of cerebrovascular events in dementia trials. CMAJ, 167(1): 1269-70, 2002.

Wooltorton E. Olanzapine (Zyprexa): increased incidence of cerebrovascular events in dementia trials. CMAJ, 170(9): 1395, 2004. 